\title{
Quantum chaos in QCD at finite temperature
}

\author{
H. Markum ${ }^{\mathrm{a}}$, R. Pullirsch ${ }^{\mathrm{a} *}$, K. Rabitsch ${ }^{\mathrm{a}}$, and T. Wettig ${ }^{\mathrm{b}}$ \\ anstitut für Kernphysik, Technische Universität Wien, A-1040 Wien, Austria

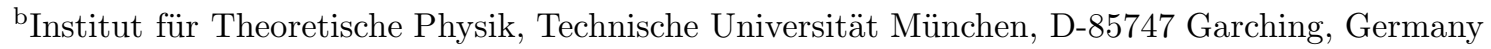

We study complete eigenvalue spectra of the staggered Dirac matrix in quenched QCD on a $6^{3} \times 4$ lattice. In particular, we investigate the nearest-neighbor spacing distribution $P(s)$ for various values of $\beta$ both in the confinement and deconfinement phase. In both phases except far into the deconfinement region, the data agree with the Wigner surmise of random matrix theory which is indicative of quantum chaos. No signs of a transition to Poisson regularity are found, and the reasons for this result are discussed.

\section{Introduction}

The fluctuation properties of the eigenvalues of the lattice Dirac operator have attracted much attention recently. In Ref. [1], it was first demonstrated that they are described by random matrix theory (RMT). In particular, the so-called nearest-neighbor spacing distribution $P(s)$, i.e., the distribution of spacings $s$ between adjacent eigenvalues, agrees with the Wigner surmise of RMT. According to a conjecture by Bohigas [2], quantum systems whose classical counterparts are chaotic have a $P(s)$ given by RMT whereas systems whose classical counterparts are integrable obey a Poisson distribution, $P(s)=e^{-s}$. In this sense, the form of $P(s)$ characterizes quantum chaos.

At the localization transition in disordered mesoscopic systems, one observes a transition in $P(s)$ from Wigner to Poisson behavior. The question of whether there is such a transition in the case of the lattice Dirac operator was first raised in Ref. [1]. The present study serves to investigate this question. Recently, a Wigner to Poisson transition was also studied in the context of a spatially homogeneous Yang-Mills-Higgs system [3].

The lattice Dirac operator falls into one of three symmetry classes corresponding to the three chiral Gaussian ensembles of RMT [4]. In Ref. [1], the Dirac matrix was studied for $\mathrm{SU}(2)$ using both staggered and Wilson fermions which corre-

\footnotetext{
*Poster presented by R. Pullirsch
}

spond to the chiral Gaussian symplectic (chGSE) and orthogonal (chGOE) ensemble, respectively. Here, we study SU(3) which for both staggered and Wilson fermions corresponds to the chiral Gaussian unitary ensemble (chGUE). We thus cover the last remaining symmetry class. The RMT result for $P(s)$ is quite complicated; it can be expressed in terms of so-called prolate spheroidal functions, see Ref. [5] where $P(s)$ has also been tabulated. A very good approximation to $P(s)$ is provided by

$P(s)=\frac{32}{\pi^{2}} s^{2} e^{-\frac{4}{\pi} s^{2}}$

which is the Wigner surmise for the chGUE.

\section{Eigenvalue analysis}

We generated gauge field configurations using the standard Wilson plaquette action for $\mathrm{SU}(3)$ and constructed the matrix of the Dirac operator using the Kogut-Susskind prescription. The Dirac matrix is anti-hermitian so that all eigenvalues are imaginary and occur in pairs with opposite sign. We have worked on a $6^{3} \times 4$ lattice with various values of $\beta$ and typically produced 10 independent configurations for each $\beta$. All spectra were checked against the analytical sum rules

$\sum_{\lambda_{n}} \lambda_{n}=0 \quad$ and $\quad \sum_{\lambda_{n}>0} \lambda_{n}^{2}=3 V$,

where $\mathrm{V}$ is the lattice volume. 
To construct $P(s)$, one first has to "unfold" the spectra [2]. This procedure is a local rescaling of the energy scale so that the mean level spacing is equal to unity on the unfolded scale. Ensemble and spectral averages (the latter is possible because of the spectral ergodicity property of RMT) are only meaningful after unfolding.

\section{Results}

Our results are displayed in the plots. Figure 1 shows the staircase function $N(E)$ which is defined as the number of eigenvalues with $\lambda \leq E$. In the continuum, $N(E)=\int_{0}^{E} \rho(\lambda) d \lambda$, where $\rho(\lambda)$ is the spectral density. Note that $\rho(\lambda)$ cannot be obtained from a random-matrix model.

Figure 2 shows the nearest-neighbor spacing distribution $P(s)$ compared with the RMT result. In the confinement phase, we find the expected agreement of $P(s)$ with the Wigner surmise of Eq. (1). In the deconfinement phase, we still observe agreement with the RMT result up to $\beta=10.0$. No signs for a transition to Poisson behavior are found. Thus, the deconfinement phase transition does not seem to coincide with a transition in the spacing distribution. For larger values of $\beta$ the eigenvalues start to approach the degenerate eigenvalues of the free theory, given by $\lambda^{2}=\sum_{\mu=1}^{4} \sin ^{2}\left(2 \pi n_{\mu} / L_{\mu}\right) / a^{2}$, where $a$ is the lattice constant and $n_{\mu}=0, \ldots, L_{\mu}-1$. In this case, the nearest-neighbor spacing distribution is trivial. However, it is possible to lift the degeneracies of the free eigenvalues using an asymmetric lattice where $L_{x}, L_{y}$, etc. are relative primes. For large lattices, the nearest-neighbor spacing distribution of the non-degenerate free eigenvalues is then given by the Poisson distribution. While it may be interesting to search for a Wigner to Poisson transition on such asymmetric lattices, it seems clear that this transition will not coincide with the deconfinement phase transition.

Furthermore, we do not think that the absence of a signature for a transition from Wigner to Poisson behavior at the deconfinement phase transition is due to the finite lattice size. Even for the small lattice size we used, the agreement of $P(s)$ with the RMT curve is nearly perfect. This leads us to believe that we should have seen some sign of a transition if it existed in the thermodynamic limit.

\section{Conclusions}

We have searched for a transition in the nearest-neighbor spacing distribution $P(s)$ from Wigner to Poisson behavior across the deconfinement phase transition. Such a transition exists, e.g., at the localization transition in disordered mesoscopic systems. In a Yang-Mills-Higgs system a smooth transition along a Brody distribution was seen [3]. We found no signature of a transition in our lattice data. The data agree with the RMT result in both the confinement and the deconfinement phase except for extremely large values of $\beta$ where the eigenvalues are trivial.

In hindsight, these results are not totally unexpected. Temporal monopole currents survive the deconfinement phase transition leading to confinement of spatial Wilson loops. Thus, even in the deconfinement phase, the gauge fields retain a certain degree of randomness. In future investigations one might try to disentangle those spatial contributions to the Dirac matrix.

\section{Acknowledgments}

This work was supported in part by FWF project P10468-PHY. We thank E.-M. Ilgenfritz, M.I. Polikarpov, and J.J.M. Verbaarschot for helpful discussions.

\section{REFERENCES}

1. M.A. Halasz and J.J.M. Verbaarschot, Phys. Rev. Lett. 74 (1995) 3920; M.A. Halasz, T. Kalkreuter, and J.J.M. Verbaarschot, Nucl. Phys. B (Proc. Suppl.) 53 (1997) 266.

2. O. Bohigas and M.J. Giannoni, Lect. Notes Phys. 209 (Springer, Heidelberg, 1984) 1.

3. L. Salasnich, Mod. Phys. Lett. A 12 (1997) 1473.

4. J.J.M. Verbaarschot, Phys. Rev. Lett. 72 (1994) 2531.

5. M.L. Mehta, Random Matrices, 2nd ed. (Academic Press, San Diego, 1991). 
Confinement: $\beta=2.8$ (solid line) and $\beta=5.0$ (dashed line)

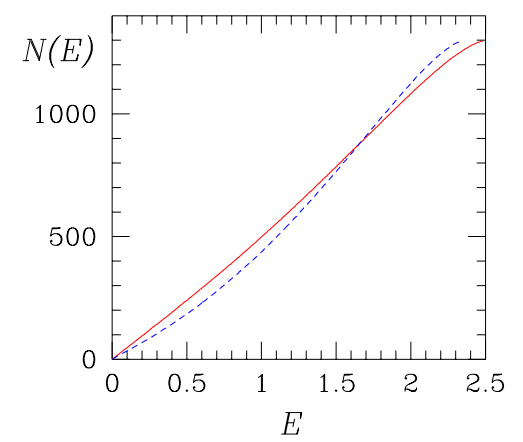

Deconfinement: $\beta=6.0$ (solid line) and $\beta=10.0$ (dashed line)

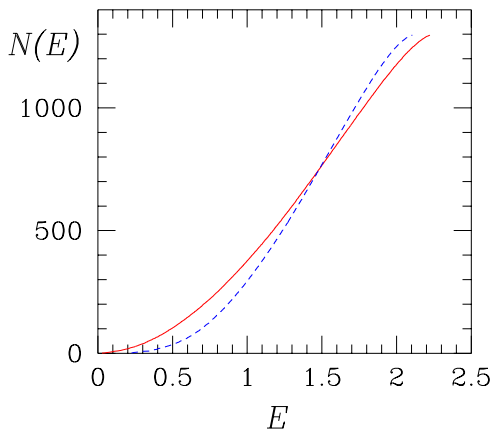

Figure 1. The staircase function $N(E)$ representing the number of positive eigenvalues $\leq E$ for typical configurations on a $6^{3} \times 4$ lattice.

Confinement: $\beta=2.8$

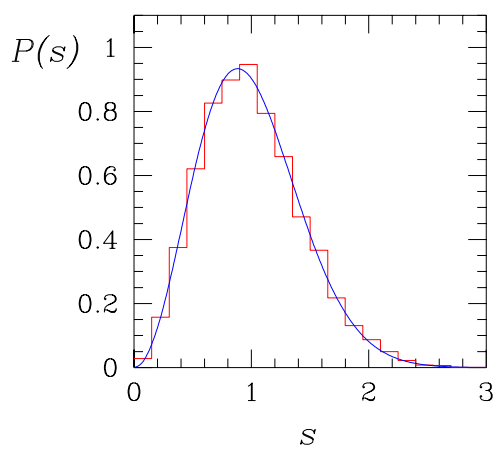

Deconfinement: $\beta=6.0$

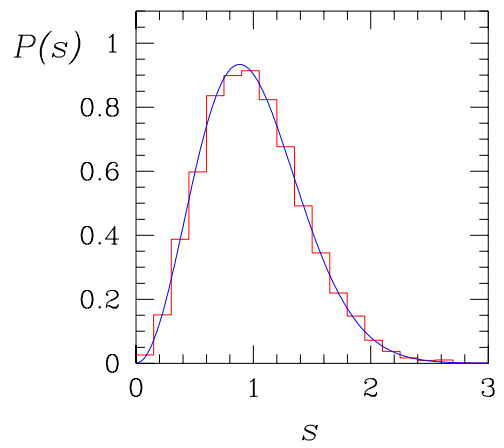

Confinement: $\beta=5.0$

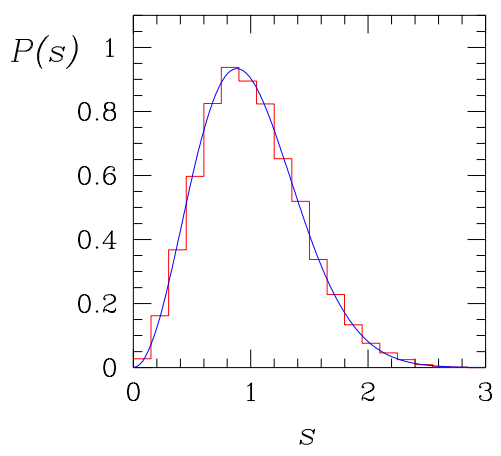

Deconfinement: $\beta=10.0$

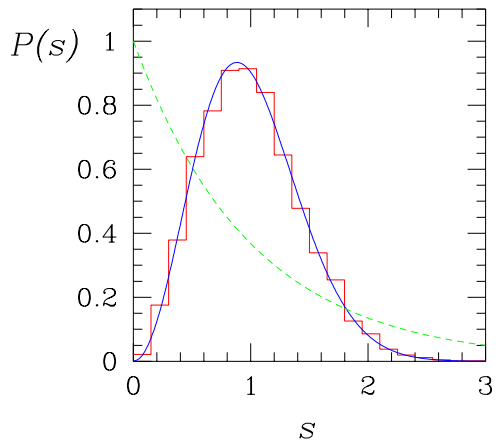

Figure 2. The nearest-neighbor spacing distribution $P(s)$ averaged over 10 independent configurations on a $6^{3} \times 4$ lattice (histograms) compared with the random-matrix result (solid lines). For comparison, the Poisson distribution $P(s)=e^{-s}$ is inserted for $\beta=10.0$ (dashed line). There are no changes in $P(s)$ across the deconfinement phase transition. 